\title{
A Mobile Health Intervention to Improve Hepatitis C Outcomes Among People With Opioid Use Disorder: Protocol for a Randomized Controlled Trial
}

Karli R Hochstatter ${ }^{1,2}$, MPH, PhD; David H Gustafson $\mathrm{Sr}^{3}$, PhD; Gina Landucci ${ }^{3}$, BS; Klaren Pe-Romashko ${ }^{3}$, MS; Adam Maus ${ }^{3}$, MS; Dhavan V Shah ${ }^{3}$, MA, PhD; Quinton A Taylor ${ }^{2}$, BS; Emma K Gill², BS; Rebecca Miller ${ }^{2}$, MSW; Sarah Krechel ${ }^{4}$, MPH; Ryan P Westergaard ${ }^{2}, \mathrm{MPH}, \mathrm{PhD}, \mathrm{MD}$

\footnotetext{
${ }^{1}$ Department of Population Health Sciences, School of Medicine and Public Health, University of Wisconsin-Madison, Madison, WI, United States

${ }^{2}$ Department of Medicine, School of Medicine and Public Health, University of Wisconsin-Madison, Madison, WI, United States

${ }^{3}$ Center for Health Enhancement Systems Studies, Department of Industrial and Systems Engineering, University of Wiconsin-Madison, Madison, WI, United States

${ }^{4}$ AIDS Resource Center of Wisconsin, Milwaukee, WI, United States
}

\section{Corresponding Author:}

Karli R Hochstatter, MPH, PhD

Department of Population Health Sciences

School of Medicine and Public Health

University of Wisconsin-Madison

1685 Highland Ave

5th Floor

Madison, WI, 53705

United States

Phone: 16082659611

Email: khochsta@medicine.wisc.edu

\section{Abstract}

Background: People who inject drugs are at a disproportionate risk for contracting hepatitis C virus (HCV). However, use of HCV prevention and treatment services remains suboptimal among people with substance use disorders due to various health system, societal, and individual barriers. Mobile health applications offer promising strategies to support people in recovery from substance use disorders. We sought to determine whether the Addiction-Comprehensive Health Enhancement Support System (A-CHESS), an existing mobile health application for opioid use disorder, could be adapted to improve HCV screening and treatment.

Objective: The goals of this paper are to describe: (1) the components and functionality of an HCV intervention incorporated into the existing A-CHESS system; and (2) how data are collected and will be used to evaluate HCV testing, linkage to care, and treatment.

Methods: People with recent opioid use were enrolled in a randomized controlled trial to test whether A-CHESS reduced relapse. We developed and implemented HCV intervention content within the A-CHESS platform to simultaneously evaluate whether A-CHESS improved secondary outcomes related to HCV care. All A-CHESS users received the HCV intervention content, which includes educational information, private messages tailored to an individual's stage of HCV care, and a public discussion forum. Data on patients' HCV risk behaviors and stage of care were collected through quarterly telephone interviews and weekly surveys delivered through A-CHESS. The proportion of people with opioid use disorder who are HCV untested, HCV-negative, HCV antibody-positive, or HCV RNA-positive, as well as linked to care, treated and cured at baseline is described here. The 24-month follow-up is ongoing and will be completed in April 2020. Survey data will then be used to assess whether individuals who received the HCV-enhanced A-CHESS intervention were more likely to reduce risky injection behaviors, receive $\mathrm{HCV}$ testing, link to medical care, initiate treatment, and be cured of HCV compared to the control group.

Results: Between April 2016 and April 2018, 416 individuals were enrolled and completed the baseline interview. Of these individuals, 207 were then randomly assigned to the control arm and 209 were assigned to the intervention arm. At baseline, 202 individuals (49\%) self-reported ever testing HCV antibody-positive. Of those, 179 (89\%) reported receiving HCV RNA confirmatory 
testing, 134 (66\%) tested HCV RNA-positive, $125(62 \%)$ were linked to medical care and $27(13 \%)$ were treated and cured of HCV. Of the remaining 214 individuals who had never tested HCV antibody-positive, $129(31 \%)$ had tested HCV antibody-negative within the past year and $85(20 \%)$ had not been tested within the past year.

Conclusions: The A-CHESS mobile health system allows for the implementation of a bundle of services as well as the collection of longitudinal data related to drug use and HCV care among people with opioid use disorders. This study will provide preliminary evidence to determine whether HCV-specific services embedded into the A-CHESS program can improve HCV outcomes for people engaged in addiction treatment.

Trial Registration: ClinicalTrials.gov NCT02712034; https://clinicaltrials.gov/ct2/show/NCT02712034

International Registered Report Identifier (IRRID)： DERR1-10.2196/12620

(JMIR Res Protoc 2019;8(8):e12620) doi: 10.2196/12620

\section{KEYWORDS}

mHealth; eHealth; hepatitis C virus; substance use; continuum of care

\section{Introduction}

In 2017, the United States Department of Health and Human Services declared a public health emergency to address the opioid crisis. This state of emergency followed the release of various national reports estimating that, in 2016, 2.1 million people had an opioid use disorder [1], 11.5 million people misused prescription opioids [1], 948,000 people used heroin [1], and 42,249 people died from overdosing on opioids [2], costing the United States approximately 504 billion dollars [3]. Among the many devastating consequences of the opioid epidemic is the increase in prevalence of hepatitis $\mathrm{C}$ virus $(\mathrm{HCV})$ through the sharing of injection equipment [4,5]. The estimated prevalence of $\mathrm{HCV}$ antibodies among people who inject drugs in the United States is 73\% [6]. According to the Centers for Disease Control and Prevention (CDC), from 2004 to 2014 acute HCV increased by $400 \%$ among people aged 18-29 and by $325 \%$ among people aged $30-39$, a trend that was highly correlated with the increase in opioid addiction treatment admissions observed during the same 11-year period [7].

Despite substantial advances in HCV treatment effectiveness and tolerability, use of prevention and treatment services remains low among people with substance use disorders due to various health system, provider, societal, and individual barriers $[8,9]$. An estimated $72 \%$ of young people who inject drugs and are living with $\mathrm{HCV}$ are unaware of their HCV infection [10], with only $31 \%$ of those diagnosed ever evaluated by an HCV specialist and only a further $8 \%$ receiving antiviral treatment [11]. Few evidence-based strategies are available for improving engagement in $\mathrm{HCV}$ care for people with a history of injection drug use.

Stigma and discrimination, lack of access to the health care system, and complex health and social needs are among the many obstacles people with substance use disorders encounter that contribute to the observed low rates of engagement in health care compared to the general population $[12,13]$. Health related smartphone applications have increased in popularity for a variety of conditions [14-16], and there is a growing evidence base suggesting that they may have a role in supporting addiction treatment. They are able to both improve access to comprehensive health services as well as make recovery support, information, and monitoring available almost constantly $[17,18]$.
The Addiction-Comprehensive Health Enhancement Support System (A-CHESS), developed at the University of Wisconsin, is a smartphone application designed to improve recovery from addiction by offering communication with peers and addiction experts, reminders and alerts to encourage therapeutic goals, individualized, addiction related, educational material, and other support services to patients. In a randomized controlled trial (RCT), A-CHESS was shown to reduce risky drinking days and enhance long term abstinence among people with alcohol use disorder, one-third of whom reported illicit drug use [18,19]. A-CHESS also reduced alcohol and opioid use in field tests with the Veterans Administration, drug courts, and among pregnant women in Appalachia [20].

Our research team previously designed and pilot tested another computerized intervention, called Hep-Net [21], to improve HCV-related outcomes among people who inject drugs and participate in harm reduction programs. The Hep-Net intervention targeted four different behavioral domains: (1) undergoing regular HCV screening; (2) using clean drug injection equipment; (3) overdose prevention; and (4) ceasing injection drug use [21]. In the context of an active RCT of A-CHESS to prevent relapse in opioid use disorder [17], our team has integrated content and functionality developed for Hep-Net into the A-CHESS platform. The goals of this substudy are to determine whether HCV-specific services embedded into the A-CHESS program can reduce risky injection behaviors and increase the frequency of HCV testing, linkage to care, and treatment for people engaged in addiction treatment. This paper describes the study protocol implemented to achieve these goals.

\section{Methods}

\section{Overall Objectives}

The goal of the parent RCT is to assess whether A-CHESS can prevent or delay relapse among people with opioid use disorder who are in early remission and receiving medication assisted treatment (MAT). Individuals with opioid use disorder from two addiction treatment centers in Massachusetts were randomly assigned in a 1:1 ratio to receive either MAT alone (control arm) or MAT and A-CHESS (experimental arm), stratifying on gender and site and balancing on age, level of care, and whether patients had prior substance use disorder treatment. Participants are followed for 24 months, a timeframe informed by prior 
research to assess the long-term impact of A-CHESS on addiction recovery with adequate power [18,22-24]. The study design, study population, recruitment, eligibility and screening process, and addiction-related services incorporated into A-CHESS have been described in the parent RCT's published protocol [17]. We developed and implemented HCV intervention content within the A-CHESS platform to simultaneously evaluate whether A-CHESS improves secondary outcomes related to $\mathrm{HCV}$ care. In this manuscript, we describe the components and functionality of the $\mathrm{HCV}$ intervention incorporated into the existing A-CHESS system and how data are collected and will be used to evaluate HCV testing, linkage to care, and treatment.

A-CHESS contains multiple services designed to address several types of challenges facing people who need treatment and prevention services for addiction. The services are organized around the components of self-determination theory, which postulates that self-motivation and well-being are a function of three innate psychological needs: competence, autonomy, and relatedness [25]. Key A-CHESS services addressing these needs include a call for help function, cognitive behavioral therapy boosters, a GPS location tracker, tailored coping support, a counselor dashboard, coach monitored discussion groups, and HIV or HCV services [17]. A-CHESS provides a medium to disseminate educational information, opportunities to interact with peers and trained counselors, and a platform for collecting participant-level data. These existing features were utilized to collect data on patients' HCV risk behaviors and testing history, and deliver behavior change interventions tailored to the patient's self-reported stage of HCV care.

A variety of study team members have administrative access to the A-CHESS system (eg, coinvestigators, project managers, research assistants and addiction counselors), allowing them to view participant data and send individualized messages. A subset of the investigator team knowledgeable about $\mathrm{HCV}$ care (hereafter referred to as $\mathrm{HCV}$ research staff) were responsible for conducting the HCV substudy.

\section{HCV Intervention Content}

\section{The HCV Care Continuum Model}

All individuals with A-CHESS received the HCV intervention discussed in this manuscript. The HCV intervention utilizes three A-CHESS components: information dissemination, private messaging, and a discussion forum. The content delivered through these components was developed in consideration of the so-called HCV care continuum: the process of how this disease is ideally managed from prevention, HCV antibody (Ab) screening, HCV RNA confirmation, undergoing HCV evaluation by a medical provider (ie, linkage to care), treatment initiation, retention in care, and then to achieving a sustained virologic response (ie, cure) [26]. Acknowledging that A-CHESS participants may fall anywhere along this continuum, each of the three $\mathrm{HCV}$ intervention components provide information and support for all stages of care, making each component relevant to the entire study population.

\section{HCV Educational Information}

Significant gaps in knowledge of HCV have been reported among people who use drugs, including a lack of knowledge regarding transmission risks, symptoms and clinical markers, as well as treatment guidelines [27-29]. Understanding that increasing knowledge of $\mathrm{HCV}$ is a critical component of public health interventions aimed at reducing the overall burden of $\mathrm{HCV}$, educational materials were incorporated into the A-CHESS system. In addition to various topics related to opioid addiction and recovery, A-CHESS houses $\mathrm{HCV}$-specific information pages that are freely navigable for users. Because injection drug use is also an important driver of the HIV epidemic, accounting for 9\% of all new HIV infections [9], A-CHESS provides HIV information as well. This educational content, housed within the information tab of A-CHESS, provides answers to frequently asked questions and links to several fact sheets developed by the CDC (Figure 1). When selecting the option Where can I get tested for HIV and Hepatitis $C$ ?, A-CHESS users are provided with a list of screening and treatment centers near their place of residence, which is determined by the site at which they enrolled into the study. Links to CDC documents and fact sheets open a Web browser and users are warned that they are leaving the A-CHESS application. To increase awareness of this information and the frequency of views, HCV research staff often reference this content and provide direct links through posts on public discussion boards and private messaging conversations.

News articles related to the intersecting epidemics of opioid injections and infectious diseases are also posted on the information tab (eg, HCV outbreaks among people who inject drugs, the release of new pharmaceutical treatments, important policy changes, and so on). Videos from people affected by these epidemics and expert medical providers working in the field are also on A-CHESS and serve as a powerful means to disseminate information to A-CHESS users. 
Figure 1. HCV and HIV educational information available on the information page of the A-CHESS app. HCV: hepatitis C virus; A-CHESS: Addiction-Comprehensive Health Enhancement Support System; CDC: Centers for Disease Control and Prevention.

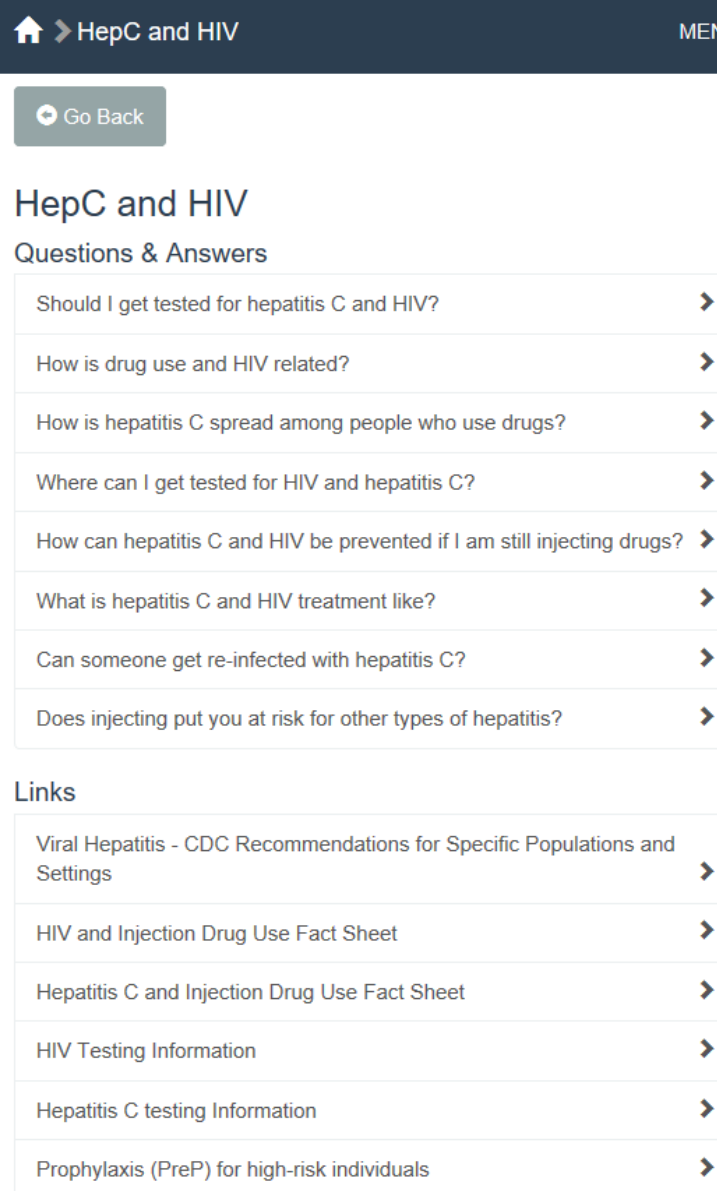

\section{Private Messaging}

Mobile health messaging interventions, such as texting, present promising strategies to increase engagement in health care. These interventions may serve a variety of purposes, including reminders, alerts, education, motivation, and prevention. A large body of literature exists that demonstrates the effectiveness of text message interventions on health outcomes, including the intent to obtain and complete the HPV vaccination series [30-32], uptake of HIV testing [33-35], improved knowledge of sexually transmitted infections (STIs) [36], and increased attendance at HIV appointments [33,37,38]. Although receiving information on HCV and other STIs through text messages has generally been well-accepted by patients [39-41], the effectiveness of mobile health messaging interventions on HCV care engagement have not been well studied. A-CHESS provides a private messaging service that functions very similarly to the standard texting application provided by smartphones, in which users may share content that is visible only to one or more intended recipients. Participants may opt in to receive automated (push) notifications on their smartphones when a private message is received in A-CHESS, when another user posts in a public discussion forum, or both.

$\mathrm{HCV}$ research staff use the private messaging feature to send messages related to goals associated with the HCV care continuum. To create a systematic approach for these private conversations, a manual was developed so that individuals in the same HCV stage of care receive the same initial message. Subsequent messages are then guided by each individual's unique response, but towards the same goal of helping each individual advance appropriately along the $\mathrm{HCV}$ care continuum. Participants received a minimum of 3 private messages from $\mathrm{HCV}$ research staff. When participants responded using the private messaging platform, the HCV research team received an automated e-mail warning them of the new message. This prompted staff to log into A-CHESS and respond at their earliest convince. When staff did not receive a response from the participant, a standardized follow-up message was sent approximately 1 month later.

Because eligibility criteria did not specify a method of opioid use, there are many participants who have not engaged in any injection risk behaviors. If an individual shares that they have never injected drugs and does not believe they are at risk of $\mathrm{HCV}$, research staff express an understanding of their low risk, share information on other means of transmission (eg, sexual contact, tattoos and piercing, and so on), encourage continued preventative actions, recommend one-time testing, and direct them to the educational content to ensure they have information to protect themselves from contracting HCV. Research staff also reference the baseline survey, discussed below, to assess each participant's date of birth. If the participant's date of birth is between 1945 and 1965, the CDC recommendation for all baby boomers to receive a one-time HCV test is shared [42]. 
Individuals who report being diagnosed with $\mathrm{HCV}$ can pose questions to $\mathrm{HCV}$ research staff who are familiar with treatment resources in their community. A coinvestigator (an infectious disease physician) maintains a user account, through which they can share health information of general interest to the group and respond to participants' nonurgent health questions related to $\mathrm{HCV}$ testing and treatment options.

\section{Discussion Boards}

Peer-based education has been shown to be an effective method for reducing risky injection behaviors and preventing HIV transmission [43-45], as well as enhancing HCV treatment knowledge and initiation $[46,47]$. A-CHESS contains several discussion boards to foster communication of different educational and emotional content. Discussion boards allow users to create and view messages that are accessible to all or only to a subset of study participants. A public group provides a forum for participants to share experiences and engage in conversations on general topics. Separate, treatment specific discussion groups allow participants to interact with other individuals receiving similar forms of MAT, including methadone, buprenorphine, or injectable naltrexone. In these groups, participants are encouraged to discuss experiences that may be specific to their form of MAT, such as side effects, dosage, and adherence challenges. A third public discussion board, named Staying Healthy, is dedicated to discussions surrounding HCV. A-CHESS users use this board to ask infectious disease related questions, share $\mathrm{HCV}$ treatment experiences, and discuss barriers to both testing and treatment. $\mathrm{HCV}$ research staff also engage in these conversations to remind A-CHESS users of the importance of being tested for HCV, encourage healthy behaviors, and stimulate discussion related to such topics. Upon study enrollment, A-CHESS users create a username of their choice, allowing them to choose an anonymous name that is unidentifiable if that makes them more comfortable when participating in these public discussions. All public discussion threads are monitored regularly by two project managers for appropriate use.

\section{Functionality for Providers}

The Moderator Dashboard provides a snapshot to study personnel of key metrics for each study participant, including their treatment center, date of enrollment, HCV status, when they last logged onto the app, A-CHESS use for the past 30 days, the past 25 private messages exchanged between the participant and study personnel, and whether or not the participant viewed the study personnel's most recently delivered private message. Private messages may also be sent directly from the moderator dashboard. Having this information readily available on one screen allows HCV research staff to quickly deliver private messages while accounting for contextual characteristics that are not displayed on the private messaging platform. Staff can use this dashboard to sort and view participants according to who they most or least recently privately messaged, who was most or least recently active on the app, and other individual characteristics. Participants can also be sorted according to their stage in the $\mathrm{HCV}$ care continuum in order to facilitate sending private messages in a systematic way. For example, an HCV research staff member could choose to only view study participants classified as $\mathrm{HCV}$-untested, and then send a private message to each individual encouraging testing.

\section{Data Collection Tools}

\section{Baseline Study Assessment and Quarterly Telephone Interviews}

Baseline questionnaires with both intervention and control participants were conducted over the phone by two study coordinators at the University of Wisconsin-Madison. Information collected at baseline included demographic items, opiate use history, chronic pain, comorbid conditions, and HCV status. Participants were asked at baseline whether they had ever been tested for $\mathrm{HCV}$, as well as the date and result of their last test. If the results from this test were positive, linkage to care and HCV treatment initiation and completion were assessed. Participants were then assigned a baseline stage of HCV care using the logic outlined in Figure 2. Time since last HCV test, whether they had injected drugs since their last test, and if they did not know the answer to questions was taken into consideration when assigning stages of $\mathrm{HCV}$ care (see Multimedia Appendix 1). Individuals were also considered $\mathrm{HCV}$-untested if they had not been tested in the past year, if they did not know whether they have ever been tested, or if they had reported injecting drugs since their last HCV test. Both intervention and control participants also received calls from the same two study coordinators quarterly, at months $4,8,12$, 16, 20 and 24. Follow-up interview questions assessed the same domains as the baseline questionnaire, allowing for the recognition of changes over time. 
Figure 2. Questions (in white boxes) asked during the baseline interview that inform the assignment of an HCV stage of care (in black boxes). "a" indicates that individuals were also considered HCV-Untested if they had not been tested in the past year, if they did not know whether they have ever been tested, or if they had reported injecting drugs since their last HCV test. HCV: hepatitis C virus; Ab: antibody.

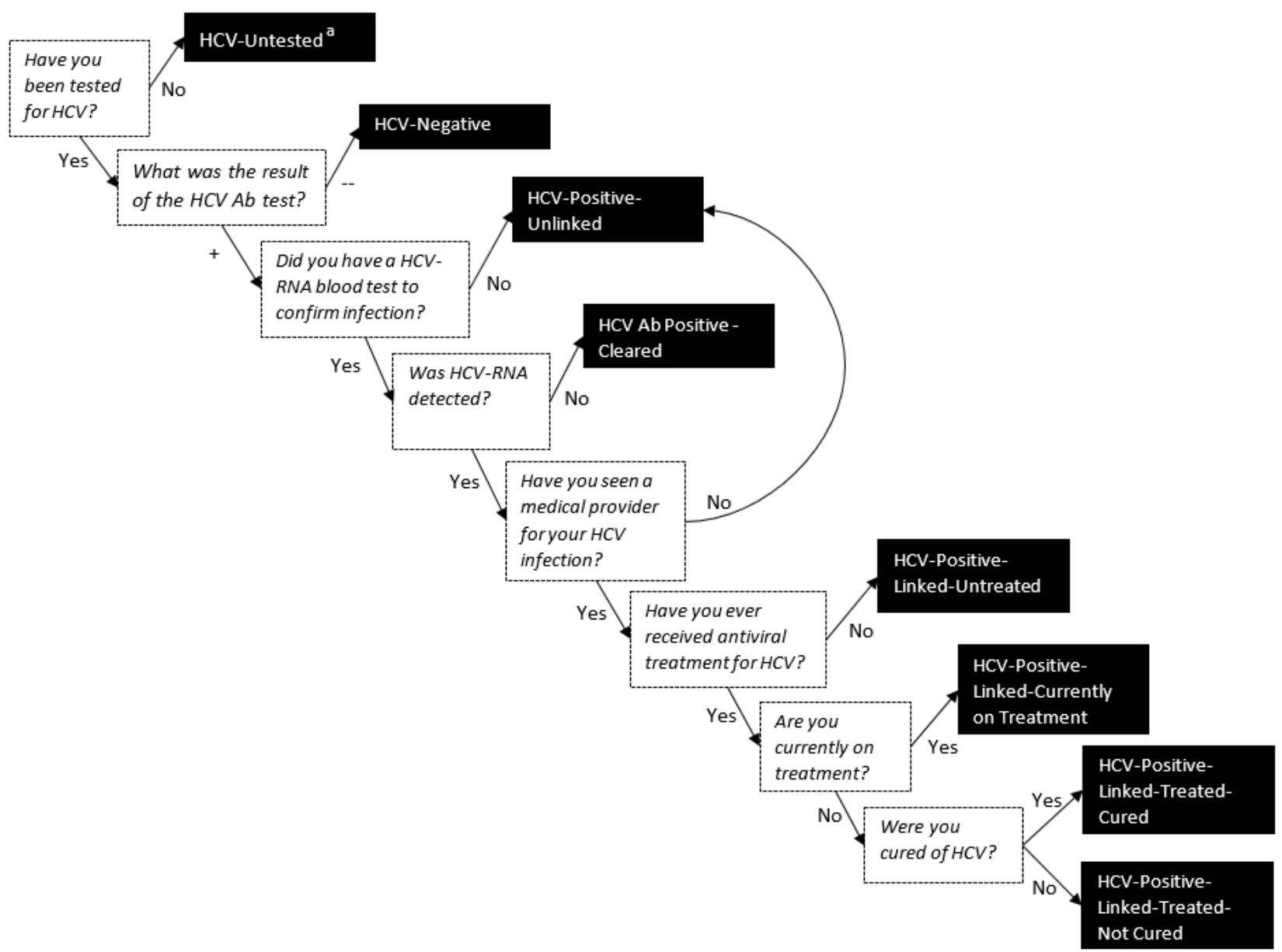

\section{Weekly Check-In Surveys}

In addition to the baseline and quarterly telephone interviews, intervention participants completed short electronic surveys delivered weekly through the A-CHESS app. The brief addiction monitor (BAM) [48], adapted for the smartphone interface using visual analog scales, provided the foundation for weekly assessments of general health status, mood, social support, MAT adherence, and progress with addiction recovery. In addition to these determinants of health, the weekly check-in captured HCV information which could be used to measure progression through the entire sequence of the HCV care continuum and the speed at which individuals received appropriate follow-up testing, got linked to medical care, initiated treatment, and were cured. Logic incorporated into the server back-end of the A-CHESS system was designed to deliver HCV-related questions for the first weekly survey according to the baseline stage of care the participants were assigned during the initial telephone interview. It then updated the HCV stage based on answers to the weekly survey (see Multimedia Appendix 2) and delivered subsequent weekly survey questions according to the newly assigned stage of $\mathrm{HCV}$ care. This logic ensured that individuals who previously tested negative for $\mathrm{HCV}$ and who continued to engage in risky injection behaviors were asked to repeat testing on a regular basis.

\section{Data Analysis}

We used data provided during the baseline telephone interviews to characterize the HCV continuum of care for all participants at the time of enrollment.

The effectiveness of this HCV intervention within the A-CHESS framework will be assessed upon completion of the 24-month follow-up. Four primary outcomes will be examined: undergoing $\mathrm{HCV}$ testing, linking to $\mathrm{HCV}$ medical care, initiating $\mathrm{HCV}$ treatment, and achieving a sustained virologic response. The quarterly telephone interviews, conducted by both intervention and control arms, allows for accurate comparisons between groups. We will conduct four separate binomial logistic regression analyses to assess whether individuals who received A-CHESS were more likely to achieve each of the primary outcomes by 24 months compared to those who did not receive A-CHESS, using an intention-to-treat approach and adjusting for the baseline stage of HCV care. The weekly survey information will allow for a more time-sensitive estimate of the rates at which individuals who use A-CHESS advance along the HCV care continuum.

Upon study completion, the frequency that different $\mathrm{HCV}$ components were utilized will also be analyzed to assess how each component is related to improved $\mathrm{HCV}$ outcomes among 
A-CHESS participants. This will allow us to understand the effectiveness of the educational information section, private messaging platform, and public discussion forums individually, as well as estimate the time elapsed between accessing different A-CHESS services and achieving HCV outcomes. Furthermore, although the weekly surveys and quarterly telephone interviews serve as data collection tools, there may be an intervention effect by reminding people to get tested, see a medical provider, and initiate and adhere to treatment. Whether these data collection tools had an intervention effect will be measured by comparing those who did and did not complete the surveys. Understanding the effectiveness of intervention components will inform future, more efficient A-CHESS models.

\section{Results}

Participant recruitment occurred during the first 2 years of the study, between April 2016 and April 2018. During this period, 416 individuals were enrolled and completed the baseline survey. Then, 207 were randomly assigned to the control group and 209 were assigned to receive A-CHESS. The 24-month follow-up of enrolled participants is expected to continue until April 2020.

At baseline, 202 individuals (49\%) reported ever testing HCV Ab-positive. Of those, 179 (89\%) reported receiving HCV RNA confirmatory testing, of which $134(75 \%)$ tested HCV RNA-positive. Among those who reported testing HCV RNA-positive, 125 (93\%) reported seeing a medical provider and $27(20 \%)$ had received HCV treatment and achieved a sustained virologic response (Table 1). Of the remaining 214 individuals who had never tested HCV Ab-positive, 129 (60\%) individuals reported testing $\mathrm{HCV}$ Ab-negative within the past year and $85(40 \%)$ reported not being tested within the past year.

Four (1\%) individuals were HIV-positive at the time of study enrollment. All four individuals were on antiretroviral medications and reported strong adherence.

Table 1. Hepatitis $\mathrm{C}$ virus continuum of care at baseline $(\mathrm{N}=416)$.

\begin{tabular}{ll}
\hline $\mathrm{HCV}^{\mathrm{a}}$ status & $\mathrm{n}(\%)$ \\
\hline Tested HCV $\mathbf{A b} \mathbf{b}^{\mathbf{b}}$-positive & $202(49)$ \\
Tested HCV RNA-positive & $134(75)$ \\
Saw a medical provider & $125(93)$ \\
Received HCV treatment & $27(20)$ \\
Not tested HCV Ab-positive & $214(51)$ \\
HCV Ab-negative & $129(60)$ \\
Not tested & $85(40)$ \\
\hline
\end{tabular}

${ }^{\mathrm{a}} \mathrm{HCV}$ : hepatitis $\mathrm{C}$ virus

${ }^{\mathrm{b}} \mathrm{Ab}$ : antibody

\section{Discussion}

\section{Overview}

The overall goal of this novel mobile health system is to support individuals recovering from opioid addiction and improve screening, linkage to care, and treatment rates for $\mathrm{HCV}$, the most common infectious disease burdening people who have a history of opioid use disorder [49]. Baseline data collected from the complete study cohort reveals that nearly half of all study participants (49\%) have tested HCV Ab-positive, while few are receiving treatment. An additional $20 \%$ of the study population had not been screened for HCV in the past year. These results demonstrate the strong need for HCV screening and treatment interventions among people with opioid use disorders and assures us that this intervention is being implemented among a population with significant potential to benefit. The overall impact that this innovative mobile health system has on HCV-related outcomes will be assessed after 24 months of follow-up interviews and weekly check-in surveys have been collected.

A prior study that examined provider and staff perceptions of A-CHESS implementation identified several facilitating factors of implementation that also served as important strategies for the implementation of the HCV intervention. Among these factors was the creation of a dedicated internal HCV team with clear roles and responsibilities to lead implementation, the orientation of clients to the content early to build awareness and interest, and the building of a separate discussion forum to stimulate conversation on $\mathrm{HCV}$ and build client engagement. These authors also highlighted the importance of collaborating with the mobile app development team to address technical issues [50]. Our close collaboration and weekly meetings with the app development team was crucial for rapidly responding to early implementation issues related to the survey logic and manually changing a participant's assigned stage of HCV care based on private messaging conversations.

\section{Limitations}

The generalizability of this study may be limited because participant recruitment was restricted to two addiction treatment centers in the state of Massachusetts. In Massachusetts, fee-for-service and managed care organizations do not have any liver damage, sobriety, or prescriber restrictions [51]. The effect of A-CHESS on HCV outcomes in states with more restricted treatment access may be limited and should be studied 
independently. Generalizability is also limited to individuals actively and newly engaged in treatment for opioid use disorder. Upon study completion, changes along the HCV care continuum can be compared across subgroups of participants receiving methadone, injectable naltrexone, and buprenorphine, but cannot be compared to individuals receiving no or other treatment regimens, those suffering from other forms of substance abuse, or those at other stages in addiction recovery.

Another limitation of this study is the use of self-reported stages of HCV care. Confusion regarding modes of transmission, the natural history of $\mathrm{HCV}$ infection, and interpretation of different $\mathrm{HCV}$ tests is common [27-29,52]. In an effort to capture these uncertainties, survey responses allowed individuals to select $I$ don't know as an answer choice to HCV questions (See the Multimedia Appendix 1).

At the time of the baseline assessment, 93\% of $\mathrm{HCV}$ RNA-positive individuals reported they had seen a medical provider for HCV, a level substantially higher than what had been reported in prior studies $[26,53,54]$. Because the survey questions did not specifically ask whether participants saw a provider specifically to discuss starting HCV treatment, our study may overestimate true linkage to HCV care. Future studies should specify details of the clinical encounter that are of interest. Fortunately, the quarterly follow-up surveys do ask if individuals have received any tests to determine whether they have evidence of liver disease, and these responses will allow us to estimate whether or not individuals received some clinical evaluation to assess their candidacy for $\mathrm{HCV}$ treatment after enrollment.

Several intrapersonal characteristics of an individual that are not measured through A-CHESS may influence engagement in care. For example, self-control, organization, and self-awareness are all facets of conscientiousness believed to influence engagement in healthcare [55]. Additionally, responses to mobile health interventions are not expected to be uniform across the study population as individuals differ significantly in their ability and willingness to engage in online communication, a construct that is difficult to measure.

\section{Conclusion}

The A-CHESS mobile health system allows for the implementation of a bundle of services and the collection of longitudinal data related to drug use and HCV care among people with opioid use disorders. This study will contribute novel data to better understand whether mobile health applications can support the complex health needs of people affected by the intersecting epidemics of opioid addiction and $\mathrm{HCV}$ infection. If effective, this mobile health application has the potential to improve HCV-related outcomes among hard to reach populations that are often disengaged from health care.

\section{Acknowledgments}

The authors acknowledge and appreciate the commitments of A-CHESS study participants and staff. This study was funded by the National Institute on Drug Abuse (R01-DA040449). KRH receives support from the Lifespan/Brown Criminal Justice Research Program on Substance Use, HIV, and Comorbidities, funded by the National Institutes of Health/National Institute on Drug Abuse (R25-DA037190).

\section{Conflicts of Interest}

DG has a shareholder interest in CHESS Mobile Health, a small business that develops Web-based health care technology for patients and family members. All other authors declare that they have no competing interests.

\section{Multimedia Appendix 1}

How to assign participants to initial HCV stage based on baseline questionnaire.

[DOCX File, 18KB-Multimedia Appendix 1]

\section{Multimedia Appendix 2}

How update HCV stages based on weekly check-in questions.

[DOCX File, 18KB-Multimedia Appendix 2]

\section{Multimedia Appendix 3}

Peer-reviewer report from the National Institute on Drug Abuse.

[PDF File (Adobe PDF File), 602KB-Multimedia Appendix 3]

\section{References}

1. Substance Abuse and Mental Health Services Administration (SAMHSA). 2017. Key substance use and mental health indicators in the United States: Results from the 2016 National Survey on Drug Use and Health URL: https://www. samhsa.gov/data/sites/default/files/NSDUH-FFR1-2016/NSDUH-FFR1-2016.htm 
2. Seth P, Scholl L, Rudd RA, Bacon S. Overdose Deaths Involving Opioids, Cocaine, and Psychostimulants - United States, 2015-2016. MMWR Morb Mortal Wkly Rep 2018 Mar 30;67(12):349-358 [FREE Full text] [doi: 10.15585/mmwr.mm6712a1] [Medline: 29596405]

3. The Council of Economic Advisers. The White House. 2017. The Underestimated Cost of the Opioid Crisis URL: https:/ /www.whitehouse.gov/sites/whitehouse.gov/files/images/

The \%20Underestimated\%20Cost $\% 20$ of\%20the\%200pioid\%20Crisis.pdf

4. Conrad C, Bradley HM, Broz D, Buddha S, Chapman EL, Galang RR, Centers for Disease Control and Prevention (CDC). Community Outbreak of HIV Infection Linked to Injection Drug Use of Oxymorphone--Indiana, 2015. MMWR Morb Mortal Wkly Rep 2015 May 01;64(16):443-444 [FREE Full text] [Medline: 25928470]

5. Centers for Disease Control and Prevention. NCHHSTP Newsroom. 2017. Increase in hepatitis C infections linked to worsening opioid crisis URL: https://www.cdc.gov/nchhstp/newsroom/2017/hepatitis-c-and-opioid-injection-press-release. $\underline{\mathrm{html}}$

6. Nelson PK, Mathers BM, Cowie B, Hagan H, Des Jarlais D, Horyniak D, et al. Global epidemiology of hepatitis B and hepatitis $\mathrm{C}$ in people who inject drugs: results of systematic reviews. Lancet 2011 Aug 13;378(9791):571-583 [FREE Full text] [doi: 10.1016/S0140-6736(11)61097-0] [Medline: 21802134]

7. Zibbell JE, Asher AK, Patel RC, Kupronis B, Iqbal K, Ward JW, et al. Increases in Acute Hepatitis C Virus Infection Related to a Growing Opioid Epidemic and Associated Injection Drug Use, United States, 2004 to 2014. Am J Public Health 2018 Feb;108(2):175-181. [doi: 10.2105/AJPH.2017.304132] [Medline: 29267061]

8. Mehta S, Genberg B, Astemborski J, Kavasery R, Kirk G, Vlahov D, et al. Limited Uptake of Hepatitis C Treatment among Injection Drug Users. Journal of community health 2008;33(3):126-133. [Medline: 18165889]

9. Centers for Disease Control and Prevention. HIV/AIDS. 2018. HIV Among People Who Inject Drugs URL: https://www. cdc.gov/hiv/group/hiv-idu.html

10. Hagan H, Campbell J, Thiede H, Strathdee S, Ouellet L, Kapadia F, et al. Self-reported hepatitis C virus antibody status and risk behavior in young injectors. Public Health Rep 2006;121(6):710-719. [doi: 10.1177/003335490612100611] [Medline: 17278406 ]

11. Iversen J, Grebely J, Catlett B, Cunningham P, Dore G, Maher L. Estimating the cascade of hepatitis C testing, care and treatment among people who inject drugs in Australia. Int J Drug Policy 2017;47:77-85. [doi: 10.1016/j.drugpo.2017.05.022] [Medline: 28578863]

12. Zeremski M, Zibbell J, Martinez A, Kritz S, Smith B, Talal A. Hepatitis C virus control among persons who inject drugs requires overcoming barriers to care. World J Gastroenterol 2013;19(44):7846-7851. [doi: 10.3748/wjg.v19.i44.7846] [Medline: 24307778]

13. Harris M, Rhodes T. Hepatitis $\mathrm{C}$ treatment access and uptake for people who inject drugs: a review mapping the role of social factors. Harm Reduct J 2013;10. [doi: 10.1186/1477-7517-10-7] [Medline: 23651646]

14. Årsand E, Frøisland D, Skrøvseth S, Chomutare T, Tatara N, Hartvigsen G, et al. Mobile health applications to assist patients with diabetes: lessons learned and design implications. Journal of diabetes science and technology 2012;6(5):1197-1206. [doi: 10.1177/193229681200600525] [Medline: PMC3570855]

15. Piette J, List J, Rana G, Townsend W, Striplin D, Heisler M. Mobile Health Devices as Tools for Worldwide Cardiovascular Risk Reduction and Disease Management. Circulation 2015;132(21):2012-2027. [doi:

10.1161/CIRCULATIONAHA.114.008723] [Medline: 26596977]

16. Free C, Phillips G, Watson L, Galli L, Felix L, Edwards P, et al. The Effectiveness of Mobile-Health Technologies to Improve Health Care Service Delivery Processes: A Systematic Review and Meta-Analysis. PLoS Medicine 2013;10(1):A. [doi: 10.1371/journal.pmed.1001363] [Medline: 23458994]

17. Gustafson DS, Landucci G, McTavish F, Kornfield R, Johnson R, Mares M, et al. The effect of bundling medication-assisted treatment for opioid addiction with mHealth: study protocol for a randomized clinical trial. Trials 2016;17(1):592. [doi: 10.1186/s13063-016-1726-1] [Medline: 27955689]

18. Gustafson D, McTavish F, Chih M, Atwood A, Johnson RA, Boyle MG, et al. A smartphone application to support recovery from alcoholism: a randomized clinical trial. JAMA Psychiatry 2014 May;71(5):566-572 [FREE Full text] [doi: 10.1001/jamapsychiatry.2013.4642] [Medline: 24671165]

19. Glass J, McKay J, Gustafson D, Kornfield R, Rathouz P, McTavish F, et al. Treatment seeking as a mechanism of change in a randomized controlled trial of a mobile health intervention to support recovery from alcohol use disorders. J Subst Abuse Treat 2017;77:57-66. [Medline: 28476273]

20. Mathews D. Evaluation Report: combatting addiction with technology for pregnant Appalachian women using smartphones. Hazard: Kentucky River Community Care, Inc; 2014.

21. Westergaard R, Hull S, Merkow A, Stephens L, Hochstatter K, Olson-Streed H, et al. Computerized Tailored Interventions to Enhance Prevention and Screening for Hepatitis C Virus Among People Who Inject Drugs: Protocol for a Randomized Pilot Study. JMIR Res Protoc 2016 22;5(1):e15 [FREE Full text] [doi: 10.2196/resprot.4830] [Medline: 26800903]

22. Cohen J. Statistical power analysis for the behavioral sciences. Hillsdale, NJ: Erlbaum Associates; 1988. 
23. McKay J, Lynch K, Shepard D, Ratichek S, Morrison R, Koppenhaver J, et al. The effectiveness of telephone-based continuing care in the clinical management of alcohol and cocaine use disorders: 12-month outcomes. J Consult Clin Psychol 2004 Dec;72(6):967-979. [doi: 10.1037/0022-006X.72.6.967] [Medline: 15612844]

24. Hedeker D, Gibbons R, Waternaux C. Sample Size Estimation for Longitudinal Designs with Attrition: Comparing Time-Related Contrasts Between Two Groups. Journal of Educational and Behavioral Statistics 2016 Aug 26;24(1):70-93. [doi: 10.3102/10769986024001070]

25. Ryan R, Deci E. Self-determination theory and the facilitation of intrinsic motivation, social development, and well-being. Am Psychol 2000 Jan;55(1):68-78. [Medline: 11392867]

26. Viner K, Kuncio D, Newbern E, Johnson C. The continuum of hepatitis C testing and care. Hepatology 2015 Mar;61(3):783-789. [doi: 10.1002/hep.27584] [Medline: 25348499]

27. O'Brien S, Day C, Black E, Dolan K. Injecting drug users' understanding of hepatitis C. Addict Behav 2008 Dec;33(12):1602-1605. [doi: 10.1016/j.addbeh.2008.07.005] [Medline: 18762384]

28. Dhopesh V, Taylor K, Burke W. Survey of hepatitis B and C in addiction treatment unit. Am J Drug Alcohol Abuse 2000 Nov;26(4):703-707. [Medline: 11097200]

29. Stein M, Maksad J, Clarke J. Hepatitis C disease among injection drug users: knowledge, perceived risk and willingness to receive treatment. Drug Alcohol Depend 2001 Feb 01;61(3):211-215. [Medline: 11164684]

30. Lee H, Koopmeiners J, McHugh J, Raveis V, Ahluwalia J. mHealth Pilot Study: Text Messaging Intervention to Promote HPV Vaccination. Am J Health Behav 2016 Jan;40(1):67-76 [FREE Full text] [doi: 10.5993/AJHB.40.1.8] [Medline: 26685815]

31. Patel A, Stern L, Unger Z, Debevec E, Roston A, Hanover R, et al. Staying on track: a cluster randomized controlled trial of automated reminders aimed at increasing human papillomavirus vaccine completion. Vaccine 2014 May 01;32(21):2428-2433. [doi: 10.1016/j.vaccine.2014.02.095] [Medline: 24631099]

32. Morris J, Wang W, Wang L, Peddecord K, Sawyer M. Comparison of reminder methods in selected adolescents with records in an immunization registry. J Adolesc Health 2015 May;56(5 Suppl):S27-S32. [doi: 10.1016/j.jadohealth.2015.01.010] [Medline: 25863551]

33. Ingersoll K, Dillingham R, Hettema J, Conaway M, Freeman J, Reynolds G, et al. Pilot RCT of bidirectional text messaging for ART adherence among nonurban substance users with HIV. Health Psychol 2015 Dec;34S:1305-1315 [FREE Full text] [doi: 10.1037/hea0000295] [Medline: 26651472]

34. de Tolly K, Skinner D, Nembaware V, Benjamin P. Investigation into the use of short message services to expand uptake of human immunodeficiency virus testing, and whether content and dosage have impact. Telemed J E Health 2012;18(1):18-23. [doi: 10.1089/tmj.2011.0058] [Medline: 22150712]

35. Mugo P, Wahome E, Gichuru E, Mwashigadi G, Thiong'o A, Prins H, et al. Effect of Text Message, Phone Call, and In-Person Appointment Reminders on Uptake of Repeat HIV Testing among Outpatients Screened for Acute HIV Infection in Kenya: A Randomized Controlled Trial. PLoS One 2016;11(4):e0153612 [FREE Full text] [doi: 10.1371/journal.pone.0153612] [Medline: 27077745]

36. Lim M, Hocking J, Aitken C, Fairley C, Jordan L, Lewis J, et al. Impact of text and email messaging on the sexual health of young people: a randomised controlled trial. J Epidemiol Community Health 2012 Jan;66(1):69-74. [doi: 10.1136/jech.2009.100396] [Medline: 21415232]

37. Bigna J, Noubiap J, Kouanfack C, Plottel C, Koulla-Shiro S. Effect of mobile phone reminders on follow-up medical care of children exposed to or infected with HIV in Cameroon (MORE CARE): a multicentre, single-blind, factorial, randomised controlled trial. Lancet Infect Dis 2014 Jul;14(7):600-608. [doi: 10.1016/S1473-3099(14)70741-8] [Medline: 24932893]

38. Joseph Davey D, Nhavoto J, Augusto O, Ponce W, Traca D, Nguimfack A, et al. SMSaúde: Evaluating Mobile Phone Text Reminders to Improve Retention in HIV Care for Patients on Antiretroviral Therapy in Mozambique. J Acquir Immune Defic Syndr 2016 Oct 01;73(2):e23-e30 [FREE Full text] [doi: 10.1097/QAI.0000000000001115] [Medline: 27632147]

39. Kaplan J, Marek H, Arya M. Patients' Attitudes Towards Text Messages about HIV and Hepatitis C Screening. JournalMTM 2018 Oct;7(2):51-54. [doi: 10.7309/jmtm.7.2.7]

40. Gold J, Lim M, Hellard M, Hocking J, Keogh L. What's in a message? Delivering sexual health promotion to young people in Australia via text messaging. BMC Public Health 2010 Dec 29;10:792-792 [FREE Full text] [doi: 10.1186/1471-2458-10-792] [Medline: 21190584]

41. Perry R, Kayekjian K, Braun R, Cantu M, Sheoran B, Chung P. Adolescents' perspectives on the use of a text messaging service for preventive sexual health promotion. J Adolesc Health 2012 Sep;51(3):220-225. [doi: 10.1016/j.jadohealth.2011.11.012] [Medline: 22921131]

42. Smith B, Morgan R, Beckett G, Falck-Ytter Y, Holtzman D, Ward J. Hepatitis C virus testing of persons born during 1945-1965: recommendations from the Centers for Disease Control and Prevention. Ann Intern Med 2012 Dec 04;157(11):817-822 [FREE Full text] [doi: 10.7326/0003-4819-157-9-201211060-00529] [Medline: 22910836]

43. Simoni J, Nelson K, Franks J, Yard S, Lehavot K. Are peer interventions for HIV efficacious? A systematic review. AIDS Behav 2011 Nov;15(8):1589-1595 [FREE Full text] [doi: 10.1007/s10461-011-9963-5] [Medline: 21598034] 
44. Go V, Frangakis C, Le Minh N, Latkin C, Ha T, Mo T, et al. Effects of an HIV peer prevention intervention on sexual and injecting risk behaviors among injecting drug users and their risk partners in Thai Nguyen, Vietnam: a randomized controlled trial. Soc Sci Med 2013 Nov;96:154-164 [FREE Full text] [doi: 10.1016/j.socscimed.2013.07.006] [Medline: 24034963]

45. Jain B, Krishnan S, Ramesh S, Sabarwal S, Garg V, Dhingra N. Effect of peer-led outreach activities on injecting risk behavior among male drug users in Haryana, India. Harm Reduct J 2014 Feb 04;11:3 [FREE Full text] [doi:

10.1186/1477-7517-11-3] [Medline: 24495379]

46. Batchelder A, Cockerham-Colas L, Peyser D, Reynoso S, Soloway I, Litwin A. Perceived benefits of the hepatitis C peer educators: a qualitative investigation. Harm Reduct J 2017 Dec 29;14(1):67-67 [FREE Full text] [doi: 10.1186/s12954-017-0192-8] [Medline: 28962652]

47. Crawford S, Bath N. Peer support models for people with a history of injecting drug use undertaking assessment and treatment for hepatitis C virus infection. Clin Infect Dis 2013 Aug;57 Suppl 2:S75-S79. [doi: 10.1093/cid/cit297] [Medline: $\underline{23884070]}$

48. Nelson K, Young K, Chapman H. Examining the performance of the brief addiction monitor. J Subst Abuse Treat 2014 Apr;46(4):472-481. [doi: 10.1016/j.jsat.2013.07.002] [Medline: 24462246]

49. Substance AAMHSA(. Medication-Assisted Treatment for Opioid Addiction in Opioid Treatment Programs: A Treatment Improvement Protocol (TIP) Series 43. Rockville, MD: U.S. Department of Health and Human Services; 2005.

50. Lord S, Moore S, Ramsey A, Dinauer S, Johnson K. Implementation of a Substance Use Recovery Support Mobile Phone App in Community Settings: Qualitative Study of Clinician and Staff Perspectives of Facilitators and Barriers. JMIR Ment Health 2016 Jun 28;3(2):e24 [FREE Full text] [doi: 10.2196/mental.4927] [Medline: 27352884]

51. National Viral Hepatitis Roundtable, Center for Health Law and Policy Innovation. Hepatitis C: The State of Medicaid Access. 2017 National Summary Report URL: https://www.chlpi.org/wp-content/uploads/2013/12/

State-of-HepC_2017_FINAL.pdf

52. Walley A, White M, Kushel M, Song Y, Tulsky J. Knowledge of and interest in hepatitis C treatment at a methadone clinic. J Subst Abuse Treat 2005 Mar;28(2):181-187. [doi: 10.1016/j.jsat.2004.12.004] [Medline: 15780548]

53. Hochstatter K, Hull S, Stockman L, Stephens L, Olson-Streed H, Ehlenbach W, et al. Using database linkages to monitor the continuum of care for hepatitis $\mathrm{C}$ virus among syringe exchange clients: Experience from a pilot intervention. Int $\mathrm{J}$ Drug Policy 2017 Dec;42:22-25 [FREE Full text] [doi: 10.1016/j.drugpo.2016.12.006] [Medline: 28126684]

54. Yehia B, Schranz A, Umscheid C, Lo Re V. The treatment cascade for chronic hepatitis C virus infection in the United States: a systematic review and meta-analysis. PLoS One 2014;9(7):e101554-e101554 [FREE Full text] [doi:

10.1371/journal.pone.0101554] [Medline: 24988388]

55. Israel S, Moffitt T. Assessing conscientious personality in primary care: an opportunity for prevention and health promotion. Dev Psychol 2014 May;50(5):1475-1477 [FREE Full text] [doi: 10.1037/a0036113] [Medline: 24773110]

\author{
Abbreviations \\ Ab: antibody \\ A-CHESS: Addiction-Comprehensive Health Enhancement Support System \\ BAM: brief addiction monitor \\ CDC: Centers for Disease Control and Prevention \\ HCV: hepatitis C virus \\ MAT: medication assisted treatment \\ RCT: randomized controlled trial \\ STI: sexually transmitted infection
}

Edited by G Eysenbach; submitted 30.10.18; peer-reviewed by H Guarino, M Nomali, E Hermes, A Ramsey; comments to author 26.02.19; revised version received 23.04.19; accepted 18.06.19; published 01.08.19

Please cite as:

Hochstatter KR, Gustafson Sr DH, Landucci G, Pe-Romashko K, Maus A, Shah DV, Taylor QA, Gill EK, Miller R, Krechel S, Westergaard $R P$

A Mobile Health Intervention to Improve Hepatitis C Outcomes Among People With Opioid Use Disorder: Protocol for a Randomized Controlled Trial

JMIR Res Protoc 2019;8(8):e12620

URL: https://www.researchprotocols.org/2019/8/e12620/

doi: $\underline{10.2196 / 12620}$

PMID: 31373273 
CKarli R Hochstatter, David H Gustafson Sr, Gina Landucci, Klaren Pe-Romashko, Adam Maus, Dhavan V Shah, Quinton A Taylor, Emma K Gill, Rebecca Miller, Sarah Krechel, Ryan P Westergaard. Originally published in JMIR Research Protocols (http://www.researchprotocols.org), 01.08.2019. This is an open-access article distributed under the terms of the Creative Commons Attribution License (https://creativecommons.org/licenses/by/4.0/), which permits unrestricted use, distribution, and reproduction in any medium, provided the original work, first published in JMIR Research Protocols, is properly cited. The complete bibliographic information, a link to the original publication on http://www.researchprotocols.org, as well as this copyright and license information must be included. 